\title{
Phyllodes Tumor of Anogenital Mammary-like Glands with Diffuse Pseudoangiomatous Stromal Hyperplasia
}

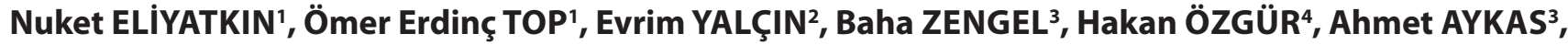 \\ Enver VARDAR ${ }^{1}$
}

Department of Pathology, 'izmir Education and Research Hospital, iZMiR, TURKEY, ${ }^{2}$ Erciş State Hospital, VAN, TURKEY,

${ }^{3}$ Department of General Surgery, İmir Education and Research Hospital, IZMIR, TURKEY, ${ }^{4}$ Department of Pathology, Güneş Pathology Laboratory, IZMiR, TURKEY

\section{ABSTRACT}

Anogenital mammary-like glands may give rise to various pathologic lesions identical to those known in mammary pathology. Tumor occurring in the anogenital region is extremely rare. The histogenetic origin of this tumor is controversial as it is being debated whether such lesions evolve from ectopic breast tissue and most recently, anogenital mammary-like gland. We report a 28-year-old girl who presented with a painless mass in the anogenital region, which was subsequently excised. Microscopic examination revealed morphologic pattern characteristic of benign phyllodes tumor with pseudoangiomatous stromal hyperplasia. We present this case to emphasize the importance of recognizing this uncommon lesion occurring at an extremely unusual site. We also discuss the histogenesis of phyllodes tumor and related lesions occurring in the anogenital region in light of the current literature along with a brief review of the previously reported cases of anogenital mammary-like glands.

Key Words: Phyllodes tumor, Pseudoangiomatous stromal hyperplasia, Female genital diseases, Perineum

\section{INTRODUCTION}

Since the first description by Hartung of mammary tissue that had developed in the vulva of a 30 -year-old female in 1872 many neoplastic and reactive conditions that are morphologically very similar to the lesions commonly seen in normal breast tissue have been described in the vulva or anogenital region (1). Such lesions include lactation glands, lactation adenoma, hydrocystoma, hydroadenoma papilliferum, non-mammary Paget disease and invasive adenocarcinoma (2). It is still unclear whether such proliferative processes in the anogenital region originate from ectopic mammary tissue or local adnexial structures. Phyllodes tumor of the breast is a relatively rare fibroepithelial lesion that makes up less than $1 \%$ of all primary breast tumors and is very rarely localized at the vulva, or the perianal or anogenital region and only a few cases have been reported to our knowledge (3-7).

We present a 28-year-old woman with benign phyllodes tumor in the perineum with widespread pseudoangiomatosis stromal hyperplasia findings. We also discuss information in the literature on the histogenesis of relevant lesions in the vulva, perineum or anogenital region, their histological features, and previously reported phyllodes tumor cases at similar localizations.

(Turk Patoloji Derg 2017, 33:157-160)

Received : 08.02.2013 Accepted : 10.08.2013

\section{CASE REPORT}

The presented case is a 28 -year-old patient who presented with a painless mass at the perineal region. The mass had a polypoid appearance and soft consistency. It measured $4.5 \times 3.5 \times 3 \mathrm{~cm}$ and was surrounded by a rim of remarkable skin. The cut surface was pale pink-white in color and in a granular fashion. Microscopy of samples showed mammary-like glands under the squamous epithelium and neighboring fibroepithelial tumor with regular borders (Figure 1). The whole tumor was processed and evaluated with multiple serial sections. Similar to the first samples, there was a marked fibroepithelial development pattern with occasional epithelial hyperplasia morphologically (Figure 2). The stroma was only mildly cellular in many areas. Stromal atypia or mitosis were absent (Figure 3). No necrosis was observed. Foci consisting of hyalinized collagen bands and slit-like spacing anastomosing with each other and lined with unspecified cells with no atypia or mitotic activity and no erythrocytes were present in the stroma (Figure 4). This specific hyalinized appearance of the stroma was interpreted as pseudoangiomatous stromal hyperplasia (PASH). There was widespread PASH around the mammary-like glands in the non-tumoral tissue. Widespread ductal hyperplasia was seen in the epithelial component. Widespread apocrine metaplasia and

Correspondence: Nuket ELIYATKIN

T.C.S.B. İzmir Eğitim ve Araştırma Hastanesi, Patoloji Bölümü,

IZMIR, TURKEY

E-mail: drnuket2003@yahoo.com Phone: +90 5064173659 
hyperplasia were also present. The tumor was quite close to the marked surgical border in some samples.

Immunohistochemical methods revealed CK7 (+), CK20 (-), CEA focal (+), GCDFP-15 focal (+), estrogen receptor $10 \%(+)$ and progesterone receptor $90 \%(+)$ in the epithelium. The stromal cells and the slits in the PASH areas stained with vimentin, actin and CD-34 (Figure 4). The slits were negative for CD31 and factor VIII.

The lesion was diagnosed as "Benign Phyllodes Tumor" with these morphological findings. Long-term follow-up for recurrence was recommended as the lesion was very close to the surgical border and it can be difficult to predict the prognosis of phyllodes tumors at such rare localizations.

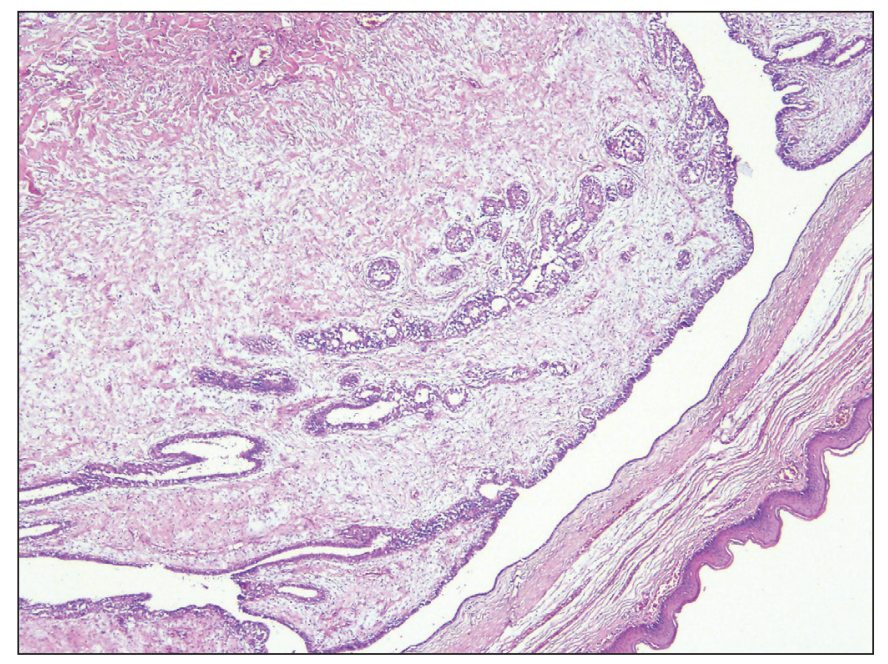

Figure 1: Circumscribed tumor with fibroepithelial development under the squamous epithelium (H\&E x40).

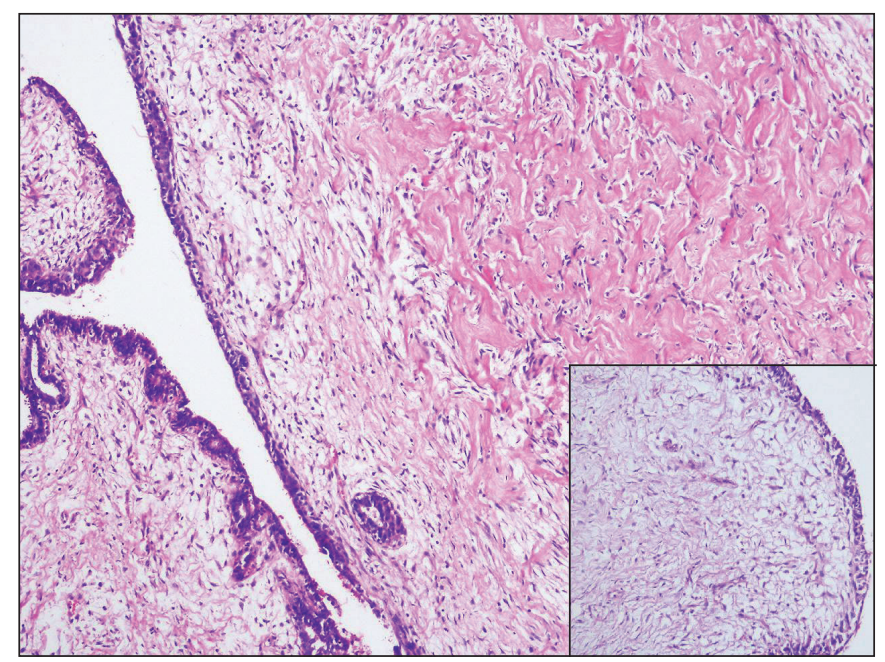

Figure 3: Normocellular and collagenized stroma (H\&E x200). Small image; no atypia and mitosis in the stromal spindle cells (H\&E x400).

\section{DISCUSSION}

Mammary-type tissue in the vulva was thought to be and accepted as ectopic mammary glands representing caudal remnants of the milk line for a long time after its description (1). The presence of ectopic breast tissue neighboring the lesion or in direct histological continuity with the lesion in some of the presented cases has also supported this notion for a long time. However, van der Putte has stated in 1991 and 1994 that this tissue represents the normal structure of the anogenital region and that similar lesions in the perianal region cannot be explained with the milk line theory $(1,8)$. Such gland structures are currently defined as anogenital mammary-like glands. van der Putte has

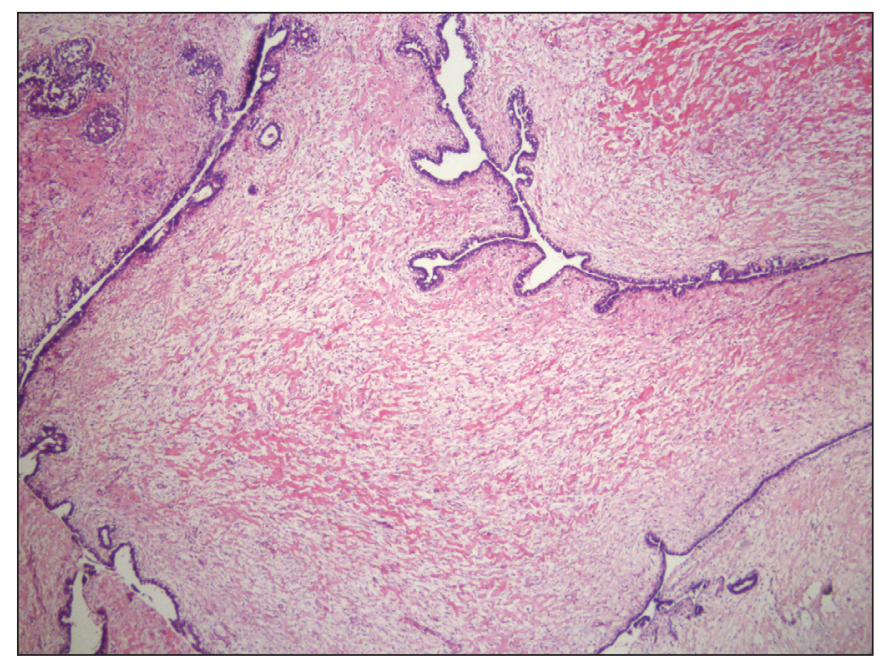

Figure 2: Typical "leaf-like” architecture (H\&E x100).

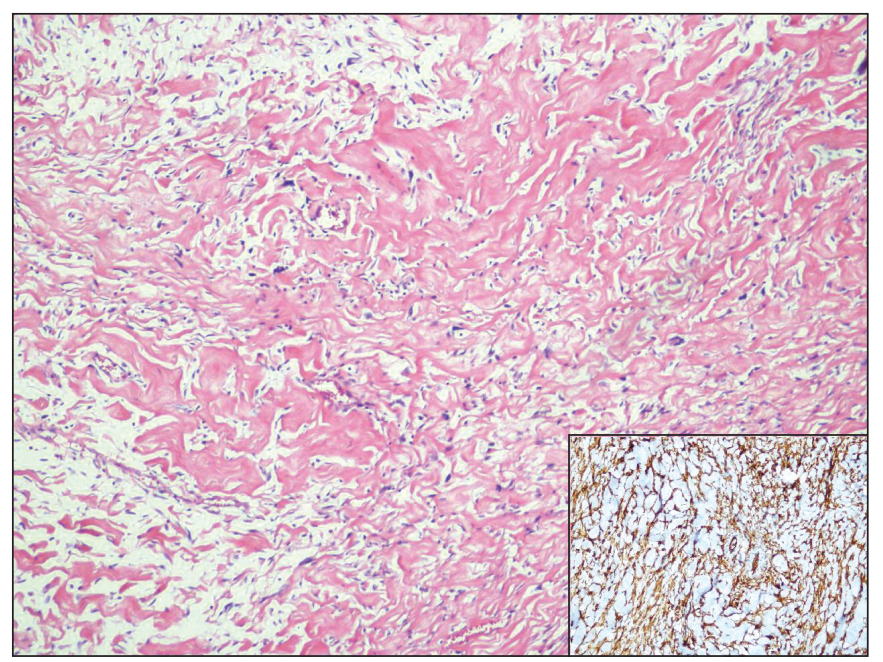

Figure 4: Close appearance of the pseudoangiomatous stromal hyperplasia areas (H\&E x200). Small image; Diffuse positivity with CD34 (CD34 x400). 
described the histological features of these lesions. Such glands are larger than apocrine or eccrine glands and have large ducti with acini, diverticuli, short branches and rarely lobules, similar to mammary glands. Ultrastructurally, the secretory epithelial cells contain electrolucent secretory granules $(1,8)$. Scurry et al have supported van der Putte's theory and recommended the use of general terminology such as "mammary-like gland adenoma" for tumors of the basophilic ductal cells of mammary-like gland structures localized in various places (9). They also believe that all of the more infrequent benign anogenital glandular tumors such as erosive adenomatosis, sclerosins adenosis, and papillary adenofibroma are variants of mammarylike gland adenomas (9). Most of these variant lesions are thought to appear in a background of typical mammarylike gland adenomas (9). The lesion was localized to the perineal region in our case and it has been confirmed that the localizations was not consistent with the milk line. This eliminates the possibility of the lesion developing from ectopic mammary tissue. Cases supporting development from ectopic mammary tissue have been reported in the literature $(2,3,4,9)$.

Our case showed mammary-like gland structures in the areas neighboring the lesion and occasionally within the lesion. We observed ductal epithelial hyperplasia, widespread apocrine metaplasia and hyperplasia and also widespread PASH in these structures and the lesion. PASH is a relatively frequent, hormone-dependent change seen with various benign and malignant processes in the breast (10). PASH has been reported to accompany various benign or malignant lesions seen in anogenital mammarylike glands (AGMLG) (10). PASH is usually a coincidental histopathological finding in the breast. However, PASH can rarely cause a clinically palpable tumor mass or massive breast enlargement. A similar situation arising in AGMLG can complicate matters (11). The presented case had ducti showing cystic dilatation and mild hyperplasia, an epithelial component with the appearance of occasionally blind ductus adenosis, and PASH with diffuse development in all stroma including that neighboring the lesion, on a morphological background of phyllodes tumor. Vazmitel et al. have defined widespread PASH in a complicated neoplasm affecting AGMLG similar to our case (11). The importance of PASH that consists of vessel-like slits is the diagnostic similarity to low-grade angiosarcoma. However, there are no erythrocytes within the vessel-like slits and atypia or mitosis in the lining epithelium. Immunohistochemistry is also helpful. Spindle cells are positive with CD34 and vimentin, partially positive with smooth muscle actin and negative with factor VIII. This immune staining pattern also indicates that the cells lining the vessel-like slits are not of endothelial origin and possibly belong to the spectrum of cells that develop from young mesenchymal cells to myofibroblasts. The fact the cells surrounding the slits were immunoreactive with CD34 and smooth muscle actin and did not stain with endothelial markers other than CD34 in our case supported a myofibroblastic origin and decreased the histopathological possibility of an angiosarcoma.

Malignant or benign, epithelial or stromal lesions of the AGMLG show significant homology with those seen in the breast (12-14). Phyllodes tumor of the AGMLG is a biphasic fibroepithelial neoplasm consisting of an epithelial glandular component and a usually more dominant stromal component. Similar to those in the breast, the definition of a neoplasm as a fibroadenoma or phyllodes tumor is based on the amount and appearance of the stromal component. The incidence of phyllodes tumor affecting the AGMLG is very low and these tumors have generally been reported as single case reports in the literature. The tumor in our case was relatively large and had fibroepithealial morphology with excess stromal development under squamous epithelium with regular borders. The entire tumor was evaluated and the stroma was mildly cellular in many areas but no stromal atypia, mitosis, heterologous differentiation or necrosis was seen. Widespread ductal hyperplasia was seen in the epithelial component, together with widespread apocrine metaplasia and hyperplasia. All features were consistent with benign phyllodes tumor. The differential diagnosis of this tumor includes some stromal tumors such as like fibromatosis, angiomyofibroblastom, angiomyxoma. The epithelial islands may look surrounded by stroma in such mesenchymal tumors but the leaf-like pattern of phyllodes tumor is not present. Papillary hydroadenoma looks like benign phyllodes tumor superficially but there is no stromal component. Two other lesions with biphasic appearance that need to be considered in the differential diagnosis are chondroid syringoma and uterine cervix mullerian adenosarcoma that has metastasized to the anogenital region. However, the characteristic "leaf-like" pattern and biphasic morphology with marked stromal component in phyllodes tumor help in the differential diagnosis with such lesions.

In conclusion, we reported an unusual case of benign phyllodes tumor with diffuse pseudoangiomatous stromal hyperplasia findings in the perineal region to highlight the current concepts regarding the origin of the tumor at the unusual site. We think that the most likely source of phyllodes tumor in anogenital region and the various other proliferative processes that show striking homology 
with lesions occurring in the normal breast is anogenital mammary-like glands. We would like to note that these lesions can be accompanied with the special morphological appearance of pseudoangiomatous stromal hyperplasia, complicating evaluation of the stromal component.

\section{REFERENCES}

1. Deaver JB, McFarland J. The breast: Its Abnormalities, Its Diseases and Their Treatment. Philadelphia, PA: Blakiston; 1917.

2. van der Putte SC. Mammary-like glands of the vulva and their disorders. Int J Gynecol Pathol. 1994;13:150-60.

3. Tbakhi A, Cowan DF, Kumar D, Kyle D. Recurring phyllodes tumor in aberrant tissue of the vulva. Am J Surg Pathol. 1993;17:946-50.

4. Tresserra F, Grases PJ, Izquierdo M, Cararach M, Fernandez-Cid. A. Fibroadenoma phyllodes arising in vulvar supernumerary breast tissue: Report of two cases. Int J Gynecol Pathol. 1998;17:171-3.

5. Chulia MT, Paya A, Niveiro M, Ceballos S, Aranda FI. Phyllodes tumor in ectopic breast tissue of the vulva. Int J Surg Pathol. 2001;9:81-3.

6. Mariappan MR, Lagera JE, Fadare O, Sibley RK. A 69-year-old woman with a vulvar lesion. Arch Pathol Lab Med. 2006;130:e11e12.

7. Giger OT, Lacoste E, Honegger C, Padberg B, Moch H, Varga Z. Expression of breast differentiation antigen NY-BR-1 in a phyllodes tumor of the vulva. Virchows Arch. 2007;450:471-4 .
8. van der Putte SC. Anogenital "sweat" glands. Histology and pathology of a gland that may mimic mammary glands. Am J Dermatopathol. 1991;13:557-67.

9. Scurry J, van der Putte SC, Pyman J, Chetty N, Szabo R. Mammary-like gland adenoma of the vulva: review of 46 cases. Pathology. 2009;41:372-8.

10. Kazakov DV, Bisceglia M, Mukensnabl P, Michal M. Pseudoangiomatous stromal hyperplasia in lesions involving anogenital mammarylike glands. Am J Surg Pathol. 2005;29:1243-6.

11. Vazmitel M, Pavlovsky M, Kacerovska D, Michal M, Kazakov D. Pseudoangiomatous stromal hyperplasia in a complex neoplastic lesion involving anogenital mammary-like glands. J Cutan Pathol. 2009: 36: 1117-20.

12. Bailey CL, Sankey HZ, Donovan JT, Beith KA, Otis CN, Powell JL. Primary breast cancer of the vulva. Gynecol Oncol. 1993;50: 379-83.

13. Baykal C, Tulunay G, Usubutun A, Küçükali T, Ozer S, Demir OF. Fibrocystic disease of vulvar ectopic breast tissue. Case report and review of the literature. Gynecol Obstet Invest. 2004;58:1514.

14. Kazakov DV, Mikyskova I, Kutzner H, Simpson RH, Hes O, Mukensnabl P, Bouda J, Zamecnik M, Kinkor Z, Michal M. Hidradenoma papilliferum with oxyphilic metaplasia: A clinicopathological study of 18 cases, including detection of human papillomavirus. Am J Dermatopathol. 2005;27:102-10. 